\title{
IDENTIFICACIÓN Y COMPARACIÓN DE SINTOMAS DE DISMORFIA MUSCULAR ENTRE SUJETOS QUE PRACTICAN FISICO CULTURISMO Y OTROS QUE REALIZAN ENTRENAMIENTO CON PESAS
}

\author{
Johnny Alberto Montoya Arroyo y Gerardo Araya Vargas \\ Escuela de Educación Física y Deportes \\ Universidad de Costa Rica, San José, Costa Rica \\ E-mail: johnny6@costarricense.cr
}

\begin{abstract}
Resumen
Montoya-Arroyo, J. A. y Araya-Vargas, G. (2003). Identificación y comparación de síntomas de dismorfia muscular entre sujetos que practican físico culturismo y otros que realizan entrenamiento con pesas. Revista de Ciencias del Ejercicio y la Salud, 3(1), 54-62. El propósito de este estudio fue identificar y comparar síntomas de dismorfia muscular, entre varones y mujeres, físico culturistas y que únicamente realizan entrenamiento con pesas. Doce físico culturistas hombres con $26.08 \pm 7.4$ años de edad; seis mujeres físico culturistas con 30.6 \pm 3.3 años de edad; catorce hombres de entrenamiento con pesas con $22.5 \pm 5.22$ años de edad; y dieciocho mujeres de entrenamiento con pesas con una edad promedio de 24.33 \pm 9.14 años, completaron la prueba Muscle Dysmorphia Scale, (MDI Scoring), así como la hoja de recolección de información general. Se realizó un análisis de covarianza, para los datos, con el que se encontró que los grupos de físico culturistas, poseen niveles significativamente más altos $(p<0.05)$ de preocupación en los factores dieta, suplementación, protección física, dependencia al ejercicio, tamaño y simetría, farmacología, en días por semana de entrenamiento y horas por sesión de entrenamiento, con respecto a los grupos de hombres y mujeres de entrenamiento con pesas. Dentro de las conclusiones más importantes, se han encontrado ciertas tendencias dismórfico corporales o vigoréxicas, entre los participantes que forman parte del grupo de físico culturistas de acuerdo con la información suministrada por los sujetos. Además, se encontraron diferencias entre varones y mujeres en algunas variables. PALABRAS CLAVES: Dismorfia muscular, vigorexia, físico culturismo, entrenamiento de contra resistencia, adicción.
\end{abstract}

\section{INTRODUCCION}

Para dar inicio en este tema, se debe recalcar que el cuerpo humano ha sido siempre un punto muy importante $\mathrm{y}$ de mucho valor a través de la historia, ya que ha estado sujeto a los patrones estéticos que dicta cada una de las diferentes culturas del mundo. Así mismo, y continuando con este proceso histórico, un hábito del ser humano moderno es el estar preocupado por su cuerpo. Hoy en día, los estereotipos implantados por las revistas de belleza y por los modelos publicitarios, han sido rápidamente acogidos por las personas, como los ideales de belleza, que demanda la sociedad moderna (Marzano, 2001; Ballone, 2001).

Como otro punto, también importante a recalcar, es que en la actualidad, se ha demostrado y documentado de manera amplia y científica los efectos beneficiosos de la práctica regular de alguna actividad física o deportiva, sobre el organismo, tanto desde un punto de vista físico como psicológico (Gulker, Laskis. y Kuba, 2001; Dishman y Jackson, 2000; Courneya y Hayduk, 2002; De Coverley, 1987; O'Connor, Raglin y Martinsen, 2000).

Ha sido así, que en respuesta a estas dos situaciones a las que se enfrenta la 
sociedad actual (apariencia o imagen física, y salud por medio de la actividad física), es que la actividad física se ha convertido no solo en un instrumento para mejorar la salud, sino también en una herramienta para obtener la imagen perfecta, es por ello que año a año se ha incrementado dramáticamente el número de personas que acuden a los gimnasios a ejercitarse; sin embargo, ¿qué sucede cuando se llega al extremo? ¿qué sucede cuando las personas caen en cierto tipo de comportamiento compulsivo o adictivo hacia la actividad física?; estas conductas han recibido diferentes constructos a través del tiempo; sin embargo, los más utilizados han sido los términos "adicción al ejercicio" o "abuso de ejercicio", debido a que no se refieren a una disciplina deportiva, ejercicio o actividad en específico, sino que se refieren de manera más general, a las personas que llevan dichas prácticas a un nivel peligroso para la salud (De Coverley, 1987; Gulker y col, 2001; Rhodes, Courneya y Hayduk., 2002).

Así mismo, estos comportamientos de adicción al ejercicio son relacionados con los trastornos dismórfico corporales, en los cuales las personas tratan de lograr una perfección física subjetiva, pues los defectos que ellos presentan, son en su mayoría imaginarios, y en algunos casos mínimos cuando son reales. Es así que estas personas toman el ejercicio como una herramienta para moldear su cuerpo, ya sea para aumentar el tamaño de la masa muscular, o por el contrario para disminuir las medidas de su cuerpo, ya que este trastorno se explica como una distorsión en la imagen corporal de sí mismo. De igual forma, se debe aclarar que para que la preocupación se considere un trastorno mental, debe causar un malestar significativo o un deterioro del funcionamiento personal, social y laboral del sujeto (Hales, Yudofsky y Talbott, 2000; Bartlett, 2001; Davis, 2000; Olivardia, 2002).

Este trastorno, (dismórfico corporal), ha sido poco estudiado, en parte debido a que es más probable que los pacientes acudan a un dermatólogo, internistas o cirujanos plásticos, antes que a un psicólogo o psiquiatra; siguiendo con el tema, en la actualidad se ha prestado poco interés a la etiología de este padecimiento, con la excepción de las relaciones sugeridas con los trastornos subyacentes del estado de ánimo, como la esquizofrenia, la fobia social o el trastorno obsesivo-compulsivo (Hales y col, 2000; de la Torre, 1995).

Sin embargo, una teoría sobre el posible origen de este fenómeno, es la que brindan, Belloch, Sandín y Ramos (1995); quienes hacen mención a las metamorfopcias, las cuales son distorsiones en la percepción visual de la forma y/o el tamaño de los objetos, ellos centran su atención en una subclasificación de estas, las autometamorfopcias, las mismas tienen una relación directa con la imagen corporal, ya que se definen como una distorsión visual en las personas para percibir su propio cuerpo, ya sea que lo hagan de manera reducida o aumentada. Otra de las teorías dada por Adams y Kirkby (2002) y Griffiths (1997), se basa en los cambios físiológicos, con el posible rol de las catecolaminas; esta hipótesis sugiere que al realizarse una actividad física, deportes o ejercicios prolongados e intensos, se contribuye en una mayor liberación de las catecolaminas, las cuales tienen un fuerte control de mando sobre el estado de ánimo, el humor, el movimiento, el sistema cardiovascular y las respuestas al estrés; por lo que altos niveles de catecolaminas se asocian con estados de ánimo positivos e inclusive sentimientos de euforia. $\mathrm{Y}$ es por eso que se considera que son estas reacciones fisiológicas y las sensaciones que brindan, las que pueden crear ciertas tendencias adictivas en el organismo de las personas, por lo que la actividad o el entrenamiento que realizan se vuelve como un tipo de droga o adictivo, y por ello la importancia que adquiere sobre otras actividades de la vida diaria.

Por otro lado, los datos actuales (Hales y col, 2000), indican que la edad de inicio de un trastorno dismórfico corporal, más frecuentemente, se podría situar entre los 
15 y los 20 años, y que la incidencia es la misma entre varones y mujeres; además esta alteración suele coexistir con otras complicaciones mentales, como trastornos depresivos, de ansiedad o de tipo psicóticos.

Dentro de la dismorfia corporal, existe una clasificación, siendo la dismorfia muscular una sub-clasificación de estas. Hablando propiamente de este trastorno, es el psiquiatra Harrison G. Pope de la Facultad de Medicina de Harvard, a quien se le atribuye el reconocimiento de este nuevo padecimiento mental, al que bautizó como vigorexia; esta se sitúa al opuesto de la anorexia, ya que las personas que presentan dismorfia muscular o vigorexia se ven excesivamente delgadas, aunque posean un cuerpo muy musculoso, y se ha encontrado principalmente entre las personas que asisten simplemente a entrenar a los gimnasios, y frecuentemente entre los que practican físico culturismo. En relación a este tema, hay otros autores que consideran que la vigorexia es un tipo de alteración de la imagen corporal y también se ha considerado como una variante del trastorno dismórfico corporal muy relacionando con un desorden obsesivocompulsivo (Matey, 1998; Baile, 2003).

Por otra parte, de acuerdo con los hallazgos del doctor Pope, citado por Matey (1998); las personas que sufren de este padecimiento (dismorfia muscular), dedican horas durante el día a su desarrollo muscular, se pesan de forma constante y siguen dietas muy estrictas, encaminadas a ganar más masa muscular y en la mayoría de las ocasiones, abusan de productos artificiales para aumentar esa masa; por tanto, estos sujetos presentan serios trastornos gastrointestinales.

Así mismo, estas personas corren el riesgo de sufrir además de ansiedad y depresión, como en el caso de los pacientes de anorexia o bulimia nerviosa, de abusar de sustancias como esteroides, anabolizantes y hormonas, los que tarde o temprano ocasionan daños en el organismo, tanto a nivel metabólico como emocional. Entre otras consecuencias que este trastorno crea en las personas, se cita que frecuentemente creen que la gente que les rodea habla y se burla de ellas; por lo cual tratan de evitar las actividades habituales, y esto en el peor de los casos, puede conducir a las personas a un aislamiento social extremo, permaneciendo en sus casas durante largo tiempo, en algunos casos hasta años. De igual forma, este tipo de trastorno puede llevar a las personas a abandonar sus estudios, evitar entrevistas profesionales y por ello mantenerse en trabajos por debajo de su verdadera capacidad o nivel, e incluso abandonar un empleo (Kaplan y Sadock, 1999). Otras características que generalmente presentan estas personas, son el tener pocas amistades, evitar las citas u otras situaciones sociales, tener problemas matrimoniales o en casos muy agudos hasta el divorcio a causa de los síntomas. Es posible que el daño o deterioro por el trastorno sea tal (aunque por supuesto que varía de un caso a otro), que conlleve a los individuaos a hospitalizaciones repetidas y en el peor de los casos a intentos suicidas (Kaplan y Sadock, 1999).

Este fenómeno se ha venido estudiando cada vez con mayor frecuencia. Por ejemplo, en el estudio de Blouin y Goldfield (1995) se comparó a 43 culturistas, 48 corredores y 48 practicantes de artes marciales, y se encontró que el grupo de los culturistas mostró niveles más altos de forma significativa que los dos grupos restantes en medidas de insatisfacción corporal, deseo de delgadez, distorsiones alimentarias y más específicamente, tendencias de tipo bulímicas, e igualmente tenían peor autoestima, aunque no diferían en cuanto a la depresión.

Como se puede observar en la literatura, es muy importante entender que se dan diferentes elementos, que pueden ser tomados como posibles factores de riesgo para desarrollar una dismorfia muscular y que su tratamiento debe ser interdisciplinario, ya que requiere la intervención de psicólogos, nutricionistas y educadores físicos. Sin embargo, el objetivo de esta investigación no se centra en el tratamiento, sino más bien, en la detección de síntomas de dismorfia 
muscular o vigorexia en personas que puedan ser posibles candidatos a desarrollar este problema, según la literatura revisada. Así mismo es importante recalcar la novedosidad de este estudio, como se ha podido observar, pues este fenómeno ha sido poco estudiado hasta ahora, y en Costa Rica mucho menos, de ahí parte de la relevancia de esta investigación.

\section{METODOLOGIA}

\section{Participantes}

Se contó con 50 sujetos, de los cuales había 18 mujeres dentro del grupo de entrenamiento con pesas, con un promedio de edad de 24.3 \pm 8.8 años; dentro del grupo de entrenamiento con pesas masculino, se contó con 14 hombres con un promedio de edad de $22.5 \pm 5.03$ años; dentro del grupo de mujeres físico culturistas participaron 6 mujeres con $30.6 \pm 3.3$ años de edad; y dentro del grupo de varones físico culturistas participaron 12 hombres, con una edad promedio de 26.08 \pm 7.16 años. Todos los participantes, asistían a gimnasios ubicados en el distrito primero del cantón central de la provincia de San José, así como del cantón de Montes de Oca.

\section{Instrumentos de medición}

Se utilizó la prueba Muscle Dysmorphia Scale (Lantz, 2003), desarrollada por Christopher Lantz (Truman University) y Deborah Rhea (Iowa State University), la cual se divide en seis subfactores: 1) Dieta, 2) Suplementación, 3) Protección Física, 4) Dependencia al Ejercicio, 5) Tamaño y simetría y 6) Farmacología. Los ítemes de cada subfactor se miden mediante una escala tipo Likert; al sumar los puntajes de los ítemes correspondientes, se obtiene el puntaje de cada subfactor. Esta escala se encuentra en estudio aún, por lo que no se cuenta con valores de confiabilidad y validez de la misma, no obstante, su creador en una comunicación personal sostenida con los autores de este trabajo (Lantz, 2003), recomendó este instrumento y dio su aval como experto para el mismo. Además, se usó una hoja de información general, elaborada por los investigadores, para recopilar información general como edad, género, tiempo de entrenar entre otras cosas.

\section{Procedimiento}

Se asistió a diferentes gimnasios ubicados en el distrito primero del cantón central de San José, así como a los del cantón de Montes de Oca. Los sujetos fueron encuestados una vez que se les administró el formulario de consentimiento informado.

\section{Análisis Estadístico}

Se utilizó como estadística descriptiva, promedios y desviaciones estándar de los puntajes obtenidos en cada uno de los factores y para cada uno de los grupos; así mismo, como estadística inferencial se aplicó un ANCOVA, tomando a la edad de los sujetos como co-variable; se encontró que existía diferencias significativas de edad entre grupos $(\mathrm{F}: 4,874 ; \mathrm{p}<0,05)$, por lo que se sospechó que esa variable podría tener un efecto en los resultados de los factores de la escala de dismorfia muscular y por eso se procedió a aplicar el ANCOVA. También se aplicó el análisis Post Hoc de Tukey, cuando fue necesario. Los datos se procesaron con el programa estadístico SPSS para Windows, versión 8.0.

\section{RESULTADOS}

Los resultados obtenidos con los respectivos análisis estadísticos, se presentan en las siguientes tablas resumen de estadística descriptiva y ANCOVA. 
Tabla \#1. Resumen de promedios y desviaciones estándar, de las distintas variables medidas en cada uno de los grupos (físico culturistas y entrenamiento con pesas; mujeres y hombres)

\begin{tabular}{lcccccccc}
\hline \multirow{2}{*}{ VARIABLE } & \multicolumn{2}{c}{ FISICOCULTURISTAS } & \multicolumn{3}{c}{ ENTRENAMIENTO PESAS } & \multicolumn{2}{c}{ TOTAL } \\
\cline { 2 - 9 } & Mujeres & Hombres & Total & Mujeres & Hombres & Total & Mujeres & Hombres \\
\hline Dieta & 23,50 & 17,5 & 19,5 & 10,72 & $10,35 \pm$ & $10,56 \pm$ & 13,91 & 13,65 \\
& $\pm 5,61$ & $\pm 5,1$ & $\pm 7,09$ & $\pm 4,7$ & 4,71 & 4,55 & $\pm 7,43$ & $\pm 6,77$ \\
Suplementación & 15,33 & 10,91 & 12,38 & 5,66 & 6,92 & 6,21 & 8,08 & 8,76 \\
& $\pm 5,57$ & $\pm 5,23$ & $\pm 5,60$ & $\pm 2,86$ & $\pm 2,78$ & $\pm 2,85$ & $\pm 5,57$ & $\pm 4,49$ \\
Protección física & 11,83 & 10,5 & 10,94 & 8,61 & 7,57 & 8,15 & 9,41 & 8,92 \\
& $\pm 5,23$ & $\pm 6,84$ & $\pm 6,22$ & $\pm 2,17$ & $\pm 2,02$ & $\pm 2,14$ & $\pm 3,38$ & $\pm 4,99$ \\
Dependencia al & 16,33 & 16,83 & 16,66 & 11,5 & 10,78 & 11,18 & 12,70 & 13,57 \\
ejercicio & $\pm 3,93$ & $\pm 5,06$ & $\pm 4,60$ & $\pm 3,38$ & $\pm 3,37$ & $\pm 3,34$ & $\pm 4,04$ & $\pm 5,16$ \\
Tamaño y & 20,66 & 19,58 & 19,94 & 8,33 & 11,42 & 9,68 & 11,41 & 13,38 \\
simetría & $\pm 3,66$ & $\pm 7,34$ & $\pm 6,25$ & $\pm 3,48$ & $\pm 4,29$ & $\pm 4,09$ & $\pm 6,45$ & $\pm 6,99$ \\
& & & & & & & & \\
Farmacología & 5,5 & 3,41 & 4,11 & 3,72 & 3,0 & 3,40 & 4,16 & 3,19 \\
& $\pm 1,76$ & $\pm 0,99$ & $\pm 1,60$ & $\pm 1,36$ & $\pm 0,00$ & $\pm 1,07$ & $\pm 1,63$ & $\pm 0,63$ \\
Edad & 30,6 & 26,08 & 28,34 & 24,3 & 22,5 & 23,4 & 27,45 & 24,29 \\
& $\pm 3,3$ & $\pm 7,16$ & $\pm 5,23$ & $\pm 8,8$ & $\pm 5,03$ & $\pm 6,91$ & $\pm 6,05$ & $\pm 6,09$ \\
Días por semana & 5,50 & 4,25 & 4,66 & 4,11 & 3,78 & 3,96 & 4,45 & 4,0 \\
de entrenamiento & $\pm 0,54$ & $\pm 0,96$ & $\pm 1,02$ & $\pm 1,02$ & $\pm 1,47$ & $\pm 1,23$ & $\pm 1,10$ & $\pm 1,26$ \\
Horas & 4,16 & 1,83 & 2,61 & 2,27 & 2,07 & 2,18 & 2,75 & 1,96 \\
entrenamiento & $\pm 0,75$ & $\pm 0,38$ & $\pm 1,24$ & $\pm 1,75$ & $\pm 1,07$ & $\pm 0,89$ & $\pm 1,11$ & $\pm 0,82$ \\
por sesión & & & & & & &
\end{tabular}

Tabla \#2. Resumen de ANCOVA, aplicados a las distintas variables dependientes para cada uno de los grupos (actividad y género)

\begin{tabular}{lccccccccc}
\hline \multicolumn{1}{c}{ VARIABLE } & \multicolumn{3}{c}{ ACTIVIDAD } & \multicolumn{3}{c}{ SEXO } & \multicolumn{3}{c}{ INTERACCION } \\
\cline { 2 - 10 } & $\mathbf{F}$ & Sig & $\mathbf{\eta 2}$ & $\mathbf{F}$ & Sig & $\boldsymbol{\eta} \mathbf{2}$ & $\mathbf{F}$ & Sig & $\boldsymbol{\eta} \mathbf{2}$ \\
\hline Dieta & $31.3^{*}$ & 0.000 & $41 \%$ & --- & --- & -- & --- & --- & --- \\
Suplementación & $32.37^{*}$ & 0.000 & $41.8 \%$ & --- & --- & -- & $5.94^{*}$ & 0.019 & $11.7 \%$ \\
Protección física & $6.01^{*}$ & 0.018 & $11.8 \%$ & --- & --- & --- & --- & --- & --- \\
Depend.. Ejercicio & $23.6^{*}$ & 0.000 & $34.4 \%$ & --- & --- & --- & --- & --- & --- \\
Tamaño y simetría & 46.88 & 0.000 & $51 \%$ & --- & --- & --- & --- & --- & --- \\
Farmacología & $8.69^{*}$ & 0.005 & $16.2 \%$ & $15.27^{*}$ & 0.000 & $25.3 \%$ & --- & --- & --- \\
Días / sem entrena. & $6.85^{*}$ & 0.012 & $13.2 \%$ & $5.16^{*}$ & 0.028 & $10.3 \%$ & --- & -- & --- \\
Hrs diarias entrena & $12.05^{*}$ & 0.001 & $21.1 \%$ & $27.6^{*}$ & 0.000 & $38 \%$ & --- & --- & --- \\
\hline
\end{tabular}

$* \mathrm{p}<0,05$

En el gráfico \#1, se observa la interacción del sexo y la actividad, mostrando una superioridad significativa en el puntaje del grupo de los físico culturistas tanto hombres como mujeres, en el factor suplementación, con respecto a los resultados obtenidos en los hombres y mujeres de entrenamiento con pesas. En este grupo no 
existieron diferencias significativas entre varones y mujeres; mientras que en el grupo de físico culturistas las mujeres presentaron una superioridad significativa respecto a los hombres de su mismo grupo de actividad, de acuerdo con el análisis de efectos simples aplicado.

Con respecto al factor protección física, se encontró que el grupo de físico culturistas, tanto hombres como mujeres, obtuvieron resultados significativamente superiores a los grupos de entrenamiento con pesas, o sea que su nivel de preocupación al respecto era mayor. Algo similar se encontró para el factor dependencia al ejercicio, donde los físico culturistas, tanto hombres como mujeres, tienen una superioridad significativa sobre los hombres y las mujeres del grupo de entrenamiento con pesas en este factor, es decir que los y las físico culturistas reportaron mayor dependencia al ejercicio.

Con relación al factor tamaño y simetría, también se presentó que los hombres y mujeres del grupo de físico culturistas, reportaron preocupaciones significativamente más altas al respecto, en comparación con los hombres y mujeres del grupo de entrenamiento con pesas.

En el factor farmacología, se encontraron diferencias según la actividad y según el sexo, pero no existió interacción significativa entre estas variables. El grupo de los y las físico culturistas, reportaron niveles más altos en este factor, que las mujeres y hombres del grupo de entrenamiento con pesas. Con respecto al sexo, tuvieron mayores puntajes las mujeres que lo hombres.

Con respecto a los días por semana dedicados al entrenamiento, aquí se da una diferencia significativamente superior por parte del grupo de los físico culturistas, sobre el grupo de entrenamiento con pesas. Así mismo, se encontró una superioridad significativa de las mujeres en comparación a los hombres, en esta variable. Es decir que en general, las mujeres invierten en promedio, más días a la semana para entrenar y en general, los sujetos que se dedican al físico culturismo, entrenan más días por semana que los que entrenan con pesas.

En el gráfico \#2, se observa la interacción en la cantidad de horas por semana dedicadas al entrenamiento, entre sexo y actividad. El post hoc mostró que las mujeres físico culturistas, tienen puntajes estadísticamente superiores que los hombres físico culturistas y que las mujeres y hombres de entrenamiento con pesas, entre los cuales no existen diferencias estadísticamente significativas entre sí.

\section{Gráfico \#1. Interación entre sexo y actividad (físico culturistas y grupo de entrenamiento con pesas) en el factor suplementación (F: $5,94 \mathrm{p}<0,05)$}

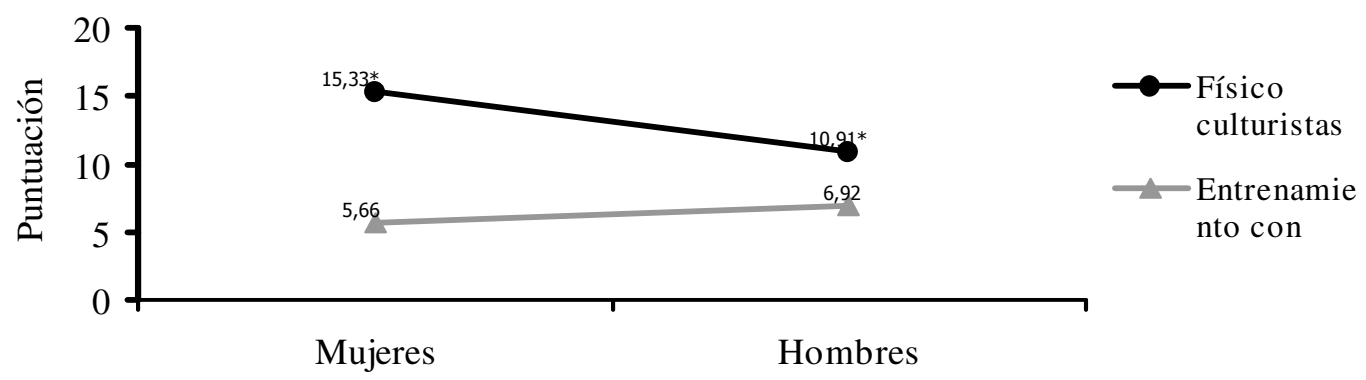




\section{DISCUSIÓN}

Los resultados que se han obtenido con este estudio, son congruentes con los encontrados en distintas investigaciones que se han desarrollado anteriormente. De acuerdo con Pope, citado por Matey (1998), los rasgos o signos de dismorfia muscular o vigorexia, se encuentran muy frecuentemente entre las personas que practican físico culturismo; así mismo, entre los datos del grupo de físico culturistas de este estudio, se encontraron resultados significativamente superiores en los factores: dieta, suplementación, protección física, dependencia al ejercicio, tamaño y simetría, farmacología, en días por semana de entrenamiento y horas por sesión de entrenamiento, con respecto a los grupos de hombres y mujeres de entrenamiento con pesas.

En cuanto al factor dieta, este explica la preocupación y el control que tiene el individuo sobre su dieta, qué tan rígida y estricta puede o no ser; aquí se encontró que tanto las mujeres como los hombres físico culturistas mostraron una preocupación significativamente superior a las mujeres y hombres participantes en el grupo de entrenamiento con pesas, en cuanto a control de su dieta; así mismo estos resultados son congruentes con los encontrados por Franco, Tamburino, Carroll y Bernal (1988), quienes estudiaron un grupo de 14 culturistas y 340 estudiantes como grupo control, y encontraron que los culturistas mostraron niveles significativamente superiores en la preocupación por la dieta y el peso corporal; por su parte Walberg y Johnston (1991), encuentran entre un grupo de 103 mujeres halterofílicas, 12 hombres culturistas y 92 mujeres estudiantes de control, que las mujeres halterofílicas mostraron actitudes de tipo patológicas sobre la alimentación e incluso incidencia de anorexia; también Blouin y Goldfield (1995), compararon un grupo de 43 culturistas, 48 corredores y 48 practicantes de artes marciales, encontrando que el grupo de los culturistas presentó tendencias de tipo bulímicas.

El factor suplementación, significa el presentar una mayor utilización de productos para suplir diferentes macro y micro nutrientes, como proteínas, fibra, vitaminas y minerales, o productos artificiales de suplementación energética, que en algunos casos, no tienen un efecto real o comprobado científicamente sobre el rendimiento. Los resultados mostraron que los físico culturistas, requieren o utilizan más frecuentemente productos para la suplementación de sustancias alimenticias, o sustancias que les ayuden a mejorar su rendimiento o para recuperarse más rápidamente durante el tiempo que presentan una lesión, en comparación con el grupo de entrenamiento con pesas, en donde no existen diferencias significativas entre los hombres y mujeres; así mismo dentro del grupo de físico culturistas son las mujeres las que presentan una utilización más frecuente de este tipo de productos.

En cuanto al factor de protección física, (insatisfacción corporal) este explica la medida en que un individuo presenta, muestra o siente cierta timidez por mostrar su físico, o exponerlo ante las personas que le rodean; así mismo este factor tiene una relación significativa con el factor tamaño y simetría, este lo que va a predecir es el nivel de preocupación que tienen las personas por su físico, más específicamente el valor que le brindan al desarrollo muscular, ya sea por deseos de ganar masa muscular o por el contrario la preocupación y molestia que ocasiona la pérdida de la misma; en estos dos factores es el grupo de mujeres y hombres físico culturistas los que presentan una superioridad estadísticamente significativa sobre las mujeres y hombres del grupo de entrenamiento con pesas, así mismo sin presentarse diferencias entre los géneros de un mismo grupo de actividad; esto se puede asociar con los resultados que obtuvieron Franco y col. (1988) donde los culturistas mostraron mayor preocupación por el peso y la figura corporal, que el grupo control; por 
su parte Blouin y Goldfield (1995) encontraron entre culturistas, niveles significativamente más altos en las medidas de insatisfacción corporal y deseo de delgadez, con respecto a un grupo de corredores y otro de artes marciales; así mismo Welberg y Johnston (1991) obtuvieron puntuaciones significativamente superiores en un grupo de halterofílicas en cuanto al deseo de delgadez, en comparación a los otros dos grupos de su estudio.

En cuanto a la dependencia al ejercicio, este factor expresa la medida de dependencia, sujeción o vínculo de las personas hacia la actividad o ejercicio que realizan; así mismo, con los resultados obtenidos en este estudio parece ser que existe una relación entre este factor y los días por semana de entrenamiento y las horas de entrenamiento por sesión, pues en ellos se marca un patrón similar en los grupos, en donde los que presentan la superioridad estadísticamente significativa, en cada una de estas variables, se mantienen constantes con respecto a los otros grupos, siendo los físico culturistas los que presentan dicha superioridad sobre los de entrenamiento con pesas; de acuerdo con Bartlett (2001), Davis (2000) y Olivardia (2002), estas conductas, que podrían indicar adicción al ejercicio, son asociadas con trastornos de dismorfia, en donde las personas toman el ejercicio como una herramienta para moldear sus cuerpos, y así alcanzar una perfección subjetiva. Por otra parte, esto podría asociarse con la teoría propuesta por Adams y Kirkby (2002) y Griffiths (1997), según la cual, de acuerdo al tipo de actividad que desarrollan, los cuerpos de estas personas realizan una mayor liberación de catecolaminas, y esto crea en ellas un cierto tipo de adicción por el ejercicio o el entrenamiento.

Con respecto al factor de farmacología, este explica la frecuencia con que el individuo requiere de la utilización de sustancias farmacológicas, como diuréticos, laxantes o esteroides; en este se encuentra que las mujeres del grupo de físico culturistas son las que muestran un nivel significativamente más alto en la utilización de este tipo de sustancias, tanto de los hombres culturistas como de las mujeres y hombres de entrenamiento con pesas; así mismo estos resultados concuerdan con los reportados por Baile (2003) y Kaplan y Sadock (1999), quienes indican que entre los problemas más frecuentes de las personas que presentan dismorfia muscular está la utilización de este tipo de sustancias, las cuales conllevan una serie de trastornos secundarios en el individuo, tanto a nivel psicológico como fisiológico.

En conclusión, se puede observar que entre el grupo de físico culturistas participantes en este estudio, existe evidencia, según sus autoreportes, de que pueden tener ciertas tendencias dismórfico musculares o vigoréxicas. Así mismo, los participantes en el grupo de físico culturismo, muestran un patrón de comportamiento similar al encontrado por otros investigadores, lo cual se asocia con las características propias de la dismorfia muscular o vigorexia. Otro aspecto importante es que existen diferencias entre varones y mujeres, en algunos de los factores estudiados, lo cual difiere un poco de lo esperado según la teoría (Hales y col, 2000); es por esta razón que es de gran importancia ahondar más en este campo, para trabajar en los posibles medios o métodos de corrección de este tipo de fenómenos, que cada vez podrían ser más frecuentes en la población costarricense que asiste a entrenar en gimnasios.

\section{BIBLIOGRAFIA}

Adams, J. y Kirkby, R. (2002) Excessive Exercise as an Addictión: A Review. Addiction Research and Theory, 10 (5), 415-437.

Baile, I. (2003) Vigorexia y culto al cuerpo, lteraciones de la imagen corporal en hombres. Revista on-line:Interpsiquis.

http://www.psiquiatria.com/imprimir.ats?9732 (5 de febrero de 2003).

Ballone, G.J (2001) Vigorexia. Documento HTML http://sities.oul.com.br/gbaloone/alimentar/vig orexia (28 may 2002).

Bartlett, J. (2001) Bigger Isn't Always Better. American Fitness, (6), 36, 37, 38 y 66. 
Belloch, A., Sandín, B. y Ramos, F. (1995) Manual de Psicopatología. (2॰ ed) Vol. 1, Madrid: Mac Graw Hill.

Blouin, A.G y Goldfield, G.S (1995) Body Image and Steroid Use in Male Bodybuilders. International Journal of Eating Disorders. 18, 159-165.

Courneya, K. y Hayduk, L. (2002) Does Personality Moderate the Theory of Planned Behavior in the Exercise Domain. Journal of Sport and Exercise Psychology, 24, 120-132.

Davis, C. (2000) Exercise Abuse. International Journal of Sport Psychology, 31, 278-289.

De Coverley, M. (1987) Exercise Dependence. British Journal of Addiction, 82, 735-740.

de la Torre, J. (1995) Mens Sana in Corpore Sano, or Exercise Abuse?: Considerations Clinics. Winter95, 59 (1), 10.

Dishman, R.K. y Jackson, E. (2000) Exercise, Fitness, and Stress. International Journal of Sport Psychology. 31, 175-203.

Franco, K.S., Tamburino, M.B., Carroll, B.T. y Bernal, G.A. (1988) Eating attitudes in college males. International Journal of Eating Disorders. 7, 285-288.

Griffiths, M. (1997) Exercise Addiction: A Case Study. Addiction Research, 5 (2), 161-168.

Gulker, M.G, Laskis, T. y Kuba, S.A. (2001) Do excessive exercisers have a higher rate of obsessive-compulsive symptomatology. Psychology, Healthy and Medicine, 6 (4), 12

Hales, R., Yudofsky, S. y Talbott, W. (2000) DSM IVTratado de Psiquiatria. ( $4^{\circ}$ ed.) París: Masson.

Kaplan, H. y Sadock, B. (1999) Sinopsis de Psiquiatría. ( $3^{\circ}$ ed.) Madrid: Panamericana.

Lantz, C. (2003) Muscle Dysmorphia Scale. Comunicación personal a través de correo eléctrónico. No disponible en la web.

Marzano, MM. (2001) The Contemporary Construction of a Perfect Body Image: Bodybuilding, Exercise Addiction and Eating Disorders. Quest, 53, 216-230.

Matey, P. (1998). Obsesionados por la perfección HTML http://www.el.mundo.es/salud/303/09N012/ (28 may 2002).

O'Connor, P., Raglin, J. y Martinsen, W. (2000) Physical Activity, Anxiety and Anxiety Disorders. International Journal of Sport Psychology, 31, 136-155.

Olivardia, R. (2002) Body Image Obsession in Men. Healthy Weight Journal, 4, 59-63.

Rhodes, R., Courneya, K. y Hayduk, L.A. (2002) Does Personality Moderate the Theory of Planned Behavior in the Exercise Domain. Journal of Sport and Exercise Psychology, 24, 120-132.

Walberg, J.L. y Johnston, C. (1991) Mestrual Dysfunctión and Eating Behaviour in Famale
Recreational Weight lifters and Competitive Body Builders. Medicine and Science in Sports and Exercise. 23, 30-36. 